\title{
Pulmonary sarcomatoid carcinoma - pathohistological and immunohistochemical analysis, prognosis and complex treatment
}

\begin{abstract}
Summary
The pulmonary sarcomatoid carcinoma (PSC) is extremely rarely lung neoplasm. A woman at the age of 55 with a local advanced pulmonary sarcomatoid carcinoma of the right lung and CT data on bilateral adrenal metastases and three brain metastases were established. Diagnosis is placed after bronchoscopy with biopsy and detailed pathochistological and immunohistochemical analysis. PSC is extremely malignant and with high risk of distant haematogenic metastases. This rare clinical case support the need for strict pathohistological and immunohistochemical analysis, a difficult pathohistological differential diagnosis with other primary malignant lung tumors and the assessment of complex treatment. In order to improve the healing results and survival of patients, timely diagnosis is required at early stage with surgical treatment and subsequent adjuvant chemotherapy and targeted therapy after genetic analysis of surgery or biopsy tissue material.
\end{abstract}

Keywords: pulmonary sarcomatoid carcinoma, pathohistological analysis, immunohistochemical analysis, hematogenic metastases, complex treatment
Volume 8 Issue 3 - 202I

\author{
Lena Marinova,' Bistra Yordanova, ${ }^{2}$ Nikolay \\ Evgeniev $^{3}$ \\ 'Department of Radiotherapy, Complex Oncology Center-Ruse, \\ Bulgaria \\ ${ }^{2}$ Clinical Pathology Department, Complex Oncology Center- \\ Ruse, Bulgaria \\ ${ }^{3}$ Department of Medical Oncology, Complex Oncology Center- \\ Ruse, Bulgaria
}

Correspondence: Lena Petkova Marinova, Department of Radiotherapy-Complex Oncology Center-Ruse, Medical University of Sofia, Bulgaria, Email rad_marinova@abv.bg

Received: June 18,2021 | Published: July 19, 2021

\section{Introduction}

The pulmonary sarcomatoid carcinoma (PSC) is a very rare malignant neoplasm, that represent $0.25 \%-1 \%$ of all lung tumors and resembles fetal lung of gestational age of 10-12 weeks. ${ }^{1,2}$ In 1952 first described by Barnard ${ }^{3}$ as an 'embryoma', and then designated as classic biphasic pulmonary blastoma (BPB) characterized by both epithelial and mesenchymal malignant components. ${ }^{4} \mathrm{PSC}$ is a unique, highly invasive pulmonary malignancy with a poor prognosis. ${ }^{5}$ Due to the rare morbidity, the histogenesis of this malignant tumor is not yet known. In this article, we will present a clinical case with PSC by trying to present the importance of immunohistochemical diagnostics of this extremely rare malignant tumor as well as a difficult pathohistological differential diagnosis with other primary lung neoplasms and the assessment of complex treatment.

Clinical case: It concerns a 55 year old woman.From 2-3 months, the patient complains of severe fatigue, easy fatigue, difficulty breathing in light physical effort, soreness in right chest half and dizziness. There is a permanent irritating cough with phlegm without blood and buzzing. For one week there is vomiting and reduced weight with 10 kilograms for a period of three months. After examination and chest radiography of the lung, a tumor process in right lung is established and is directed to fibrobronchoscopy.

Bronchoscopy with biopsy: vocal cords and traches: Without pathological changes. Tracheal section - free. The right upper lobe bronchus is completely trimmed by a tumor with necrosis, heavily bleed spontaneously and in manipulations. A biopsy spicimen for histology was obtained. Conclusion - Tumor of the right lung.

CT of a brain with venous contrast: Annular lesion subtitled to the right cerebellum up to $40 \mathrm{~mm}$ and supratentorial on the right temporal and temporo-occipital with a maximum size of $25 \mathrm{~mm}$. Conclusion Three brain metastases (Figure 1).
CT of thorax and abdomen with venous contrast: A solid tumor up to $14 \mathrm{~cm}$ in the greatest diameter situated in the right upper pulmonary lobe. Without parenchymal pulmonary pathology to the left. Liver normal dimensions and parenchymal structure, without $\mathrm{CT}$ data on metastases. Adrenales - metastatic tumor enlarged double-sided in a right with dimensions up to $11 \mathrm{~cm}$. No pathologically increased mesenteric, paraaortic and inguinal lymph nodes. No CT data on bone metastases or free fluid in the abdomen and the small pelvis (Figure 2).

Patohistological morphology: Histological examination revealed classical malignant biphasic growth pattern with poorly defined tubular structures lined by single or more layers of hyperchromatic cells (undifferentiated epithelial component), surrounded by undifferentiated mesenchymal tissue, consisting of oval-to-spindle shaped cells with hyperchromatic nuclei (mesenchymal component). Cells are visualized variable cellularity with varied size, as well as giant cells usually bizarre with multilobulated nuclei and with irregular mitosis resemble a muscle tumor, histiocyte-like cells with vacuolated nuclei, atypical cells with hyperchromatic nuclei, irregular fascicles, pleomorphic and bizarre tumor cells with foamy cytoplasm and marked atypia in background of collagenous stroma (Figure 3, Figure 4).

Immunohistochemistry (IHC): P63- Focal positive IHC expression in single epithelial undifferentiated tumor cells; TFT1-Focal positive IHC expression in the epithelial tumor component; Vimentin - Strong positive expression in sarcomoid tumor cells, and Vimentin negative nests from undifferentiated epithelial cell component; CK 5/6Negative IHC expression and barely noticeably positive expression in single undifferentiated epithelial cells at a large field zoom x100); CK7- Strong positive expression in epithelial cell component; CK AE1-AE3- Strong positive expression in epithelial cell component (Figures 5-7). 


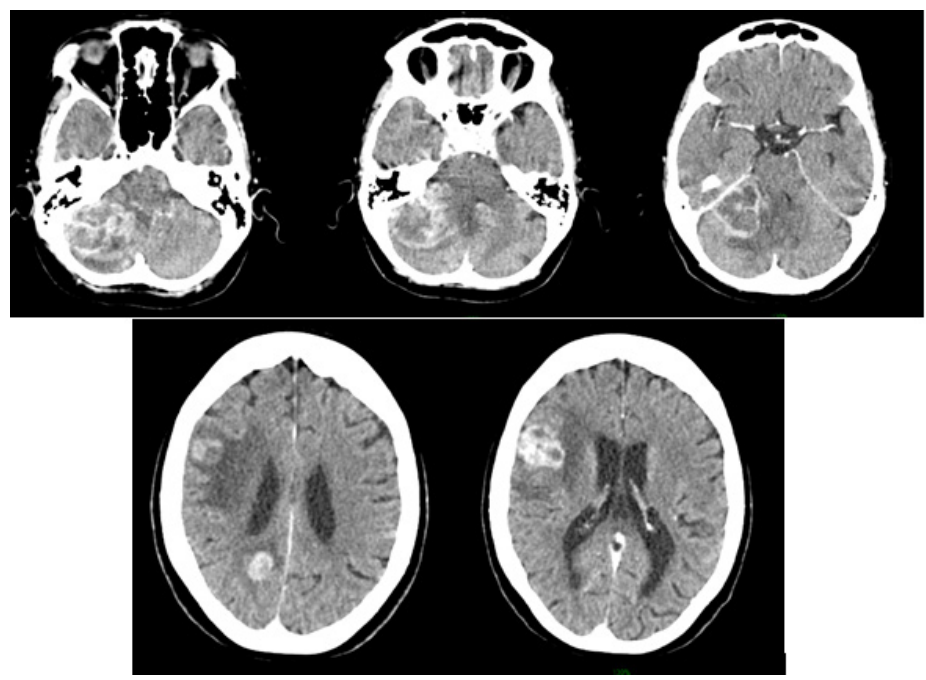

Figure I AX CT scans with intravenous contrast-Three major contrast accumulating brain metastases are visualized, one of whom is in the cerebellum and the other two supratineal in the right brain hemisphere.

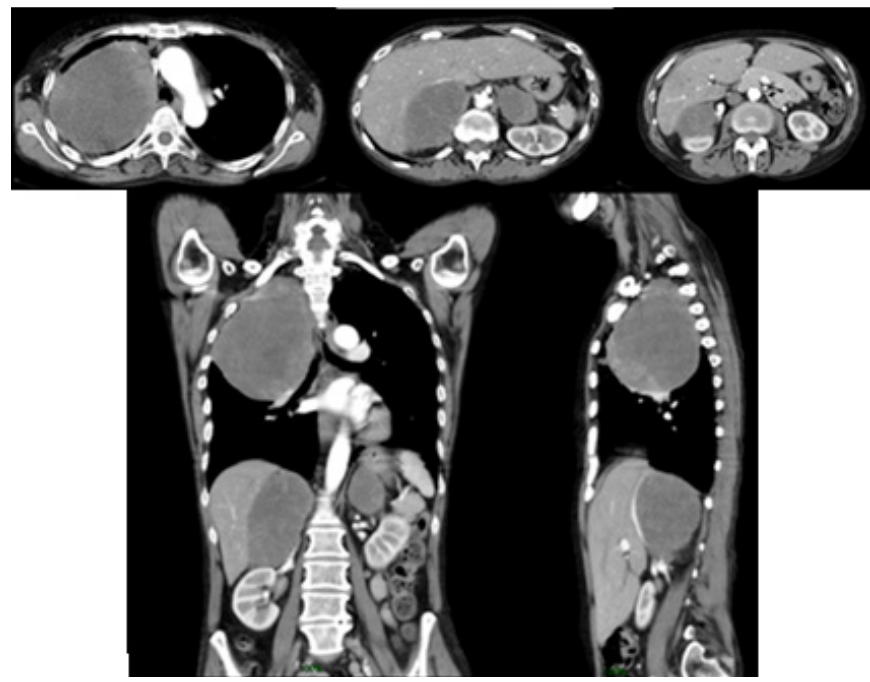

Figure 2 AX; COR and SAG CT scans with intravenous contrast- The well-circumscribed pulmonal mass, covering over $60 \%$ of the right lung parenchyma with a maximum size of $14 \mathrm{~cm}$, as well as right adrenal metastasis with a maximum size of $\mathrm{II} \mathrm{cm}$ and left adrenal metastasis with a maximum size of $5 \mathrm{~cm}$.

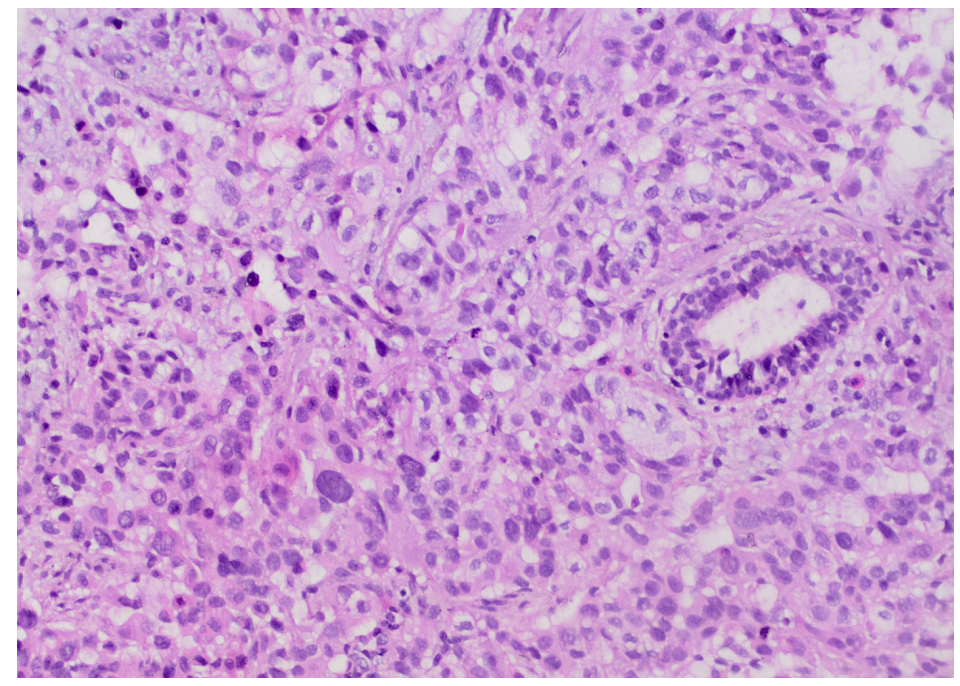

Figure 3 Photomicrography from biopsy of pulmonary tumor visualized cells with varied size and oval to spindle shape, as well as giant cells with irregular mitosis, histiocyte-like cells with vacuolated nucleus, atypical cells with hyperchromatic nuclei (representing a mesenchyme components) and tubular structures, lined by single and multiple layers of hyperchromatic cells (representing an epithelial component) $(\mathrm{H}$ and $\mathrm{E}, \times 100)$. 


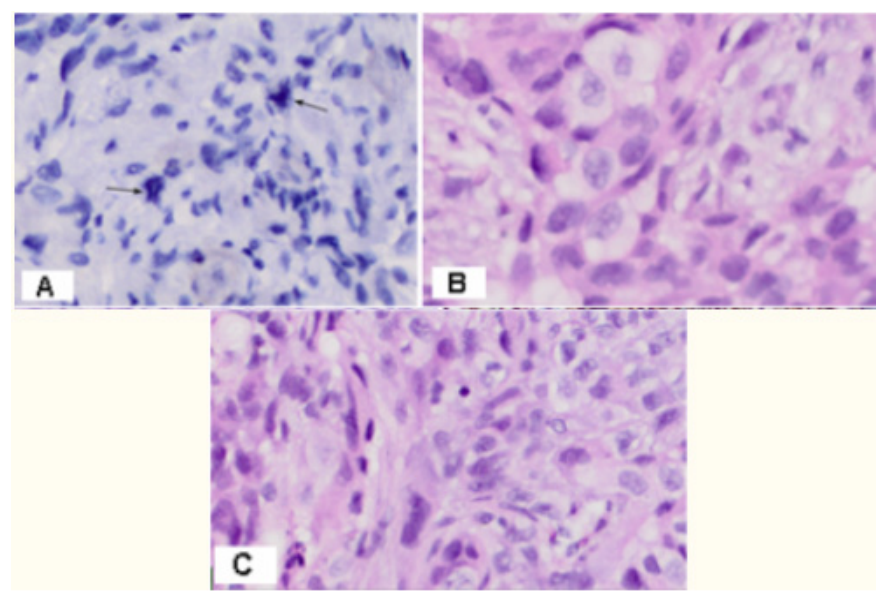

Figure 4 Photomicrography from biopsy of pulmonary tumor visualized (A) Giant cells with irregular mitosis $x 40$; B/ Histiocyte-like cells with vacuolated nucleus $\times 100 ; \mathrm{C} /$ Irregular fascicles, variable cellularity, pleomorphic and bizarre tumor cells with foamy cytoplasm and marked atypia, in background of collagenous stroma, giant cells usually bizarre with multilobulated nuclei resemble a muscle tumor $\times 40$.

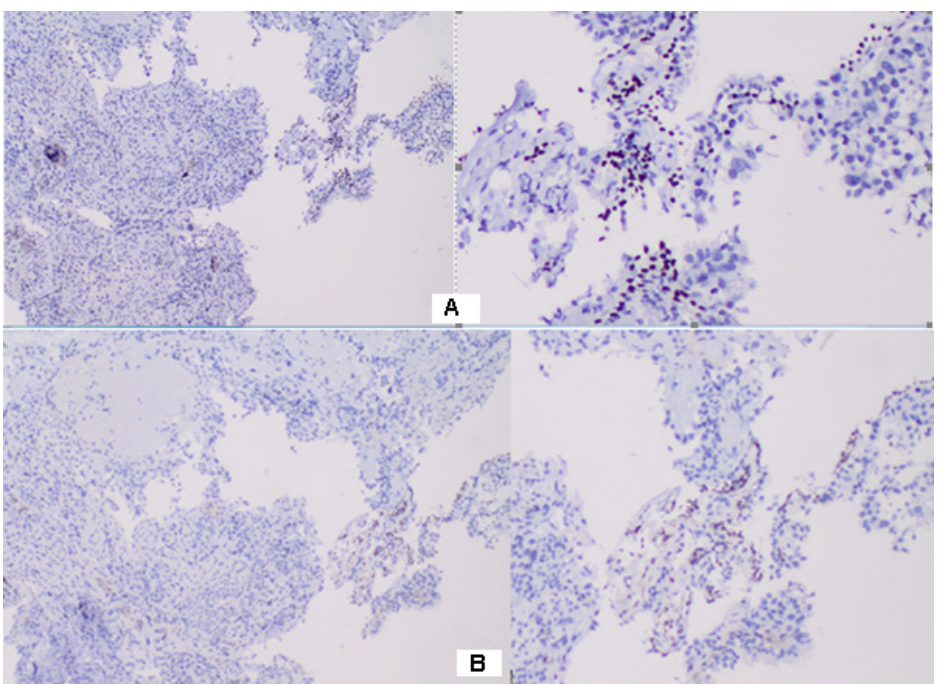

Figure 5 Immunohistochemistry- A / p63- Focal positive IHC expression in single epithelial tumor cells x20; x100; B/ CK 5/6- Negative IHC expression and weak positive in single undifferentiated epithelial cells $\times 20$; barely noticeably positive single cells at a large field zoom $\times 100$.

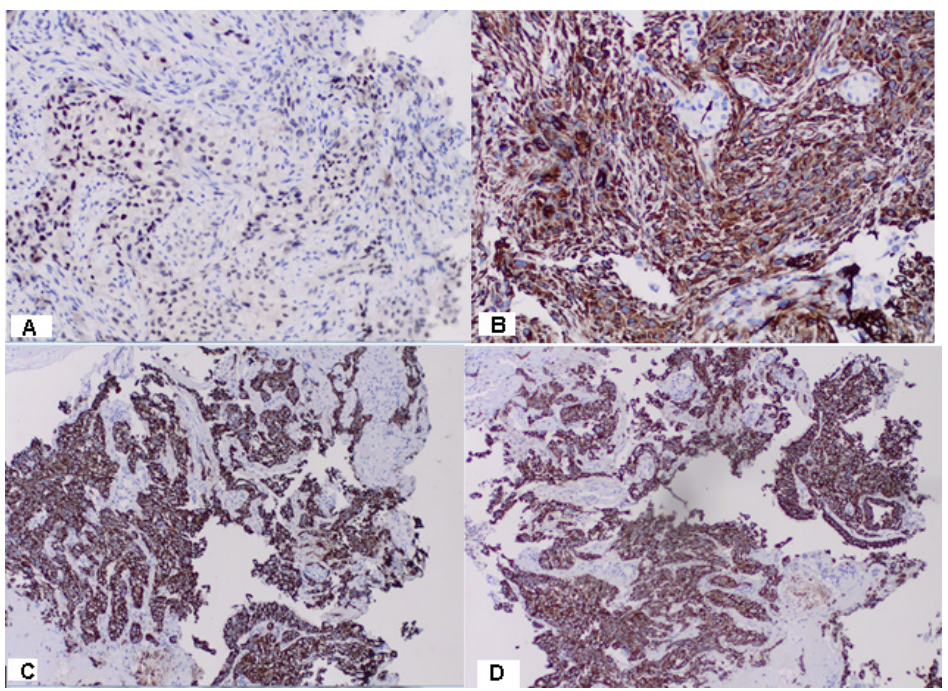

Figure 6 Immunohistochemistry-A/ TFTI- Focal positive IHC expression in the epithelial tumor cells x20; B/ Vimentin - Strong positive expression in sarcomoid tumor cells, and Vimentin negative nests from undifferentiated epithelial cell component x20; C/ CK7- Strong positive expression in epithelial cell componen x20t; D/ CK AE I-AE3- Strong positive expression in epithelial cell component x20.

Citation: Marinova L, Yordanova B, Evgeniev N. Pulmonary sarcomatoid carcinoma - pathohistological and immunohistochemical analysis, prognosis and complex treatment. J Lung Pulm Respir Res. 2021;8(3):86-92. DOI: 10.15406/jlprr.2021.08.00257 


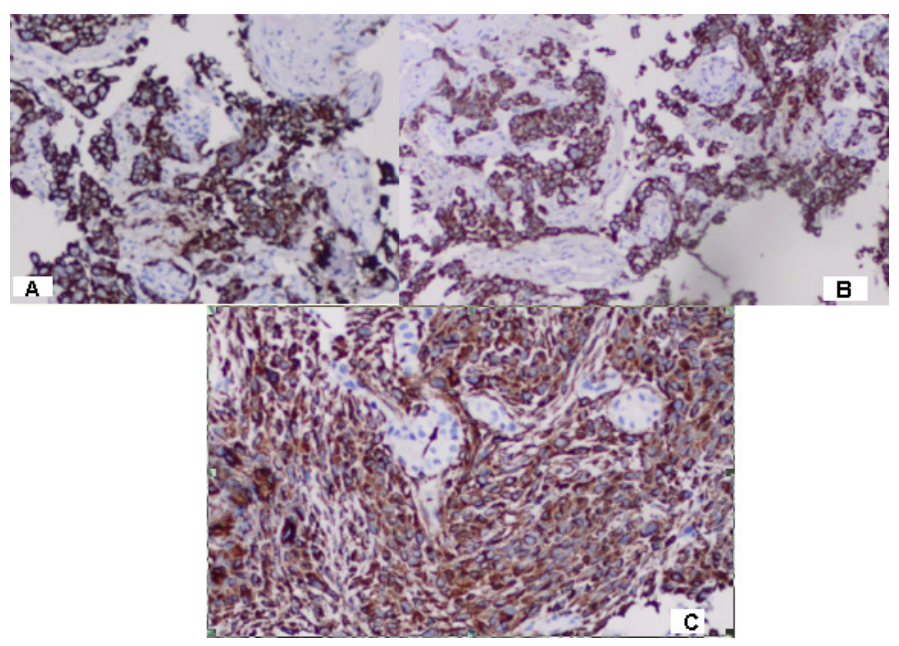

Figure 7 Immunohistochemistry-A/CK7 and B/ CKAE I-AE3- Strong positive expression in epithelial cell component and negative nests with sarcomoid tumor cells xI00 C/Vimentin - Strong positive expression in sarcomoid tumor cells and negative nests with undifferentiated epithelial cell component xI00.

Based on patohistological morphology and immunohistochemical analysis, we determine the final histological diagnosis- biphasic malignant tumor/ combination of undifferentiated carcinoma with a pleomorphic sarcoma or the so-called pulmonary sarcomatoid carcinoma.

The clinical case was addressed and discussed on oncological commission, which reccommended radiosurgery for brain metastases, followed with chemotherapy and targeted therapy in the absence of contraindications after a genetic study of the histological material.
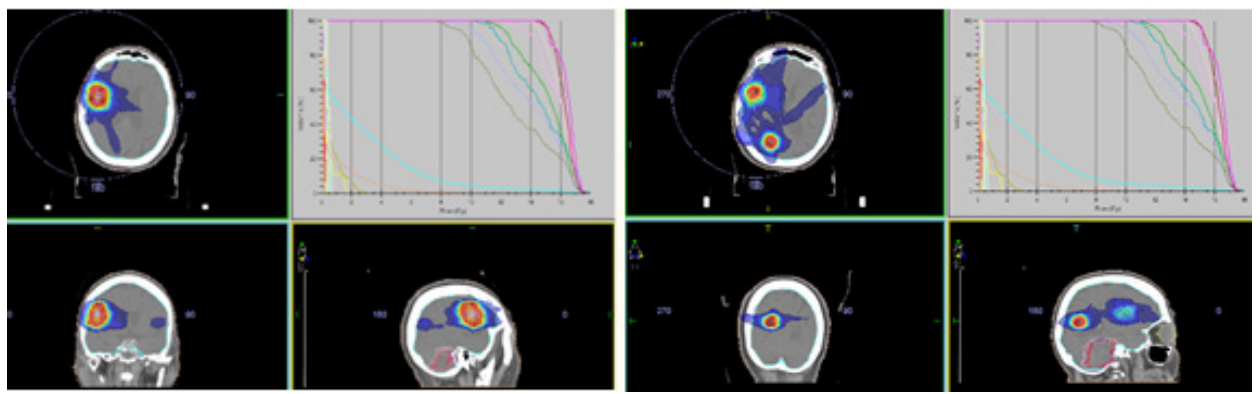

Figure 8 Intensity modulated radiotherapy (IMRT) / radiosurgery (RS) with VMAT technique in the field of the two brain metastases in the right brain hemisphere with a daily dose (DD) 15 Gy / one fraction.
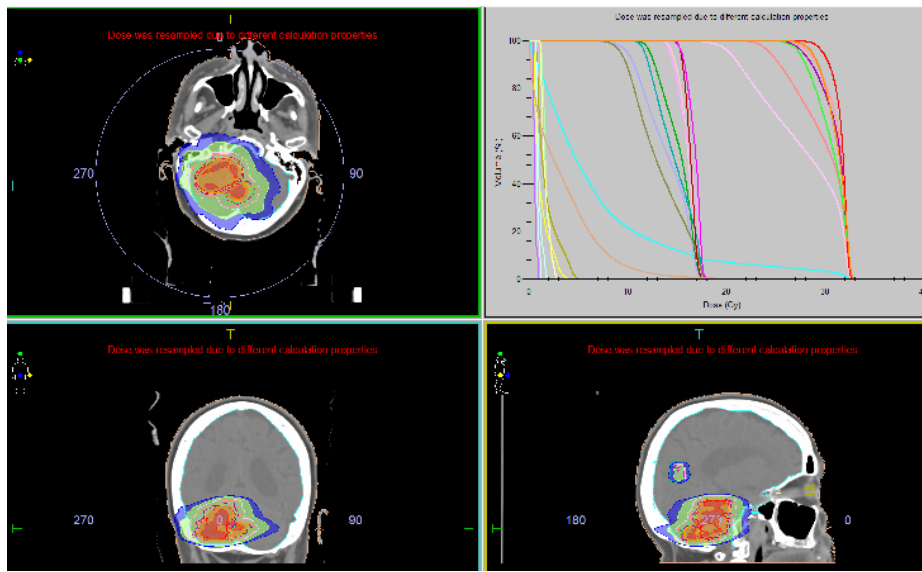

Figure 9 Intensity modulated radiotherapy (IMRT) by hypofractionated RS/ three fractions with DD 10 Gy up to TD 30 Gy of the two metastases in the cerebellum, that are merged into a single target.

Citation: Marinova L,Yordanova B, Evgeniev N. Pulmonary sarcomatoid carcinoma - pathohistological and immunohistochemical analysis, prognosis and complex treatment.J Lung Pulm Respir Res. 2021;8(3):86-92. DOI: 10.15406/jlprr.2021.08.00257 


\section{Discussion}

Sarcomatoid carcinoma is a rare form of cancer in which the cells exhibit properties characteristic of both epithelial and mesenchymal tumors. It can occur in multiple organs, including skin, bone, thyroid, breast, liver, pancreas, urinary tract, and lung. ${ }^{6}$

The primary pulmonary sarcomatoid carcinoma (PSC) is a very rare malignant neoplasm, that represent $0.25-0.5 \%$ of all primary lung tumors. ${ }^{1}$ PSC is with distinctive pathological features, clinical course, and prognosis. ${ }^{7}$ The histogenesis is exactly not known whether the tumor arises only from the endoderm or endoderm and mesoderm. It is also not clear whether; rather benign looking blastoma and frank carcinosarcoma really belong to one group as two ends of spectrum or represent entirely different lesion. ${ }^{8}$

Clinical characteristic and image diagnostics: PSC has a variable clinical course that cannot be determined by its histological appearance. ${ }^{9}$ Classical PLSC usually presents with nonspecific respiratory symptoms such as cough, hemoptysis, dyspnea, and chest pain. ${ }^{4}$ On CT thorax, the lesion often shows solid components with necrotic areas as seen in our case. The size of the lesion may be variable, with a reported average size of about $10 \mathrm{~cm} .{ }^{4}$ From 2-3 months, our patient complains of severe fatigue, easy fatigue, difficulty breathing in light physical effort, soreness in right chest half and dizziness. There is a permanent irritating cough with phlegm without blood and buzzing. There is vomiting and weight reduction about 10 kilograms for a period of three months. We observed a rapid growth tumor with hematogenic metastases in the both adrenal glands and in the brain. The radiological appearance consisted of the well-circumscribed giant malignant pulmonal mass, covering over $60 \%$ of the right lung parenchyma with a maximum size of $14 \mathrm{~cm}$, as well as right adrenal metastasis with a maximum size of $10 \mathrm{~cm}$ and left adrenal metastasis with a maximum size of $5 \mathrm{~cm}$ (Figure 2). Metastases are spread in the brain. On a CT with intravenous contrast, three major metastases are visualized, one of whom is in the cerebellum and the other two supratineal in the right brain hemisphere (Figure 1). One month after diagnostic cerebral $\mathrm{CT}$, the localization $\mathrm{CT}$ for planning radiosurgery visualized another metastasis in the cerebellum located near the previous one. This finding takes into account the rapid dynamics of the spread of this rare tumor.

Prognosis: Sarcomatoid carcinoma is very aggressive, with an overall 5-year survival rate of approximately $20 \% .{ }^{10}$ The adverse prognostic indicators are tumor size more than $5 \mathrm{~cm}$, metastatic disease at initial presentation. ${ }^{11}$ The prognosis is usually poor and about two-thirds of patients die within 2 years of diagnosis. ${ }^{4,12}$ PSC had a median survival of 10 months, which was much shorter than for other lung carcinomas (20 months for adenocarcinoma, 12.6 months for large cell carcinoma, and 18.5 months for squamous cell carcinoma). ${ }^{13}$ Sarcomatoid carcinoma is characterized by rapid growth, invasion, disease recurrence, and metastases. ${ }^{12}$ Metastatic PSC involves the same organ sites that are affected by more usual forms of lung cancer: namely, the opposite lung, liver, bones, adrenal glands, and brain. ${ }^{14}$ In clinical cases with a large inoperable primary lung tumor with distant adrenals and brain metastases, the forecast is poor as it is an extremely radio/chemoresistance tumor cells. In our clinical case, only radiotherapy for brain metastases was possible.

Patohistological morphology: The definition of the World Health Organization (WHO) for Pleomorphic Carcinoma is subtype of sarcomatoid carcinoma; usually aggressive, malignant epithelial neoplasm composed of cells with significant cytologic atypia and nuclear pleomorphism, that contains at least $10 \%$ spindle cells and/ or giant cells which can present as one or a mixture of the two cell types. ${ }^{7,15,16}$ The primary lung pleomorphic carcinoma (PLPC) is included under the recent WHO classification of "carcinomas with pleomorphic, sarcomatoid, or sarcomatous elements". In 31-72\% of PLPC cases, an adenocarcinoma cell component, in $12-26 \%$ cases with squamous cell component and in $43 \%$ cases undifferentiated epithelial cell component is detected. Sarcomatoid carcinoma may consist of a heterogeneous group of non-small cell carcinomas that also contain a component of true sarcoma, or they may be composed in part or completely of sarcoma-like elements. ${ }^{12}$ Pleomorphic sarcomatous elements composed of fibroblasts, myofibroblasts and histiocyte-like cells. The name Pleomorphic cells means variation in cytoplasmic and nuclear size throughout the tumor, not just funny looking cells. ${ }^{17}$ Cells are spindled, plasmacytoid and pleomorphic (often multinucleated), all with malignant nuclear morphology. ${ }^{18}$ For definitive diagnosis, multiple trucut lung biopsies were done that on histological examination revealed classical malignant biphasic growth pattern with well-defined tubular structures lined by single or more layers of hyperchromatic cells (epithelial component) surrounded by undifferentiated mesenchymal tissue consisting of oval-tospindle shaped cells with hyperchromatic nuclei (mesenchymal component). ${ }^{4}$ In the presented clinical case, all cellular characteristics of the sarcomatoid cell component include storiform pattern (cells emanate from a central focus), irregular fascicles, variable cellularity, pleomorphic and bizarre tumor cells with foamy cytoplasm and marked atypia in background of collagenous stroma with zones of cell necrosis (Figure 3,4).

Immunohistochemistry: (IHC)- Several studies have evaluated sarcomatoid carcinoma by IHC using Keratin antibodies and/or electron microscopy. These studies concluded that the spindle cell component of the neoplasm was of epithelial derivation. ${ }^{19}$ In sarcomatoid cell component positive IHC expression to CK7 (63\%), TTF1 (43\%), Surfactant Protein A (6\%), ${ }^{7}$ Vimentin, CD10 and CD68 is reported. ${ }^{17}$ In epithelial cell component positive expression to CK7 (76\%), TTF1 (59\%), Surfactant Protein A (39\%) and negative expression to CK20 is reported. ${ }^{7}$ On immunohistochemistry, the glandular component was positive for cytokeratin and epithelial membrane antigen (EMA), while the pleomorphic stromal component was positive for Vimentin and Smooth Muscle Antigen (SMA). ${ }^{4}$ Immunohistochemical stain p63 $(91 \%)$ and CK $5 / 6(90 \%)$ are sometimes $(9 \%)$ positive in squamous cell carcinoma and are rarely $(4 \%)$ positive in adenocarcinoma $(\mathrm{P}<.001) .{ }^{20}$ Sarcomoid carcinoma (SC) expresses the following epithelial markers P63, P40, Pankeratin and thyroid transcription factor-1 (TTF1). ${ }^{21,22}$ All three immunohistochemical markers of epithelial differentiation including p63, MOC-31, and TTF1 were quite specific for epithelial differentiation on SCs. ${ }^{23}$ Strong cytoplasmic expression of CD117 in both mesenchymal and epithelial cells also suggests a single origin and supports the idea that PLPC arises from a pluripotential cell, that can differentiate into both stromal and epithelial morphologies. ${ }^{24}$ p63 appears to be expressed in stem cells and highly-proliferating transit-amplifying cells of ectodermal origin. However, it is present at low levels or absent in differentiating cells as well as cells of mesenchymal origin. ${ }^{25}$ The $\mathrm{Np} 63 \alpha$, the predominant p63 isoform in stratified squamous epithelium, is localized to the basal cells and is overexpressed in squamous cell cancers of multiple organ sites, including skin, head and neck, and lung. ${ }^{26}$ On immunohistochemistry in the presented clinical case, the epithelial cell component (undifferentiated epithelial cells) was focal positive for P63 and TFT1, strong positive for CK7 and cytokeratin (CK AE1-AE3) and negative for $\mathrm{CK} 5 / 6$, while the pleomorphic stromal component was strong positive for Vimentin (Figures 5-7). Interesting is Figure 7, which shows the immunohistochemistry with epithelial and 
mesenchymal markers. It highlights outbreaks from mesenchymal sarcomatoid cells negative to epithelial markers and those of the epithelial component negative to mesenchymal markers. In this way, the bifactic morphology of this rare tumor is well seized.

Differential diagnosis (DD): Sarcomatoid carcinomas can be difficult to distinguish from large cell carcinoma and pulmonary sarcomas. ${ }^{27} \mathrm{DD}$ was imposed with primary pulmonary pleomorphic sarcoma, lacking expression to IHC markers of epithelial differentiation. It is difficult to differentiate sarcomatoid carcinomas (SCs) from undifferentiated pleomorphic sarcoma. Stronger Cytokeratin immunoreactivity along with more differentiated carcinomatous elements, as well as immunoreactivity to other epithelial markers (such as P63) can be helpful in the diagnosis of SCs..$^{21}$ Neural tissue, mesenchymal tissue tumours, cutaneous lymphomas and histiocytosis, Paget's disease, and atypical fibroxanthoma lack p63 in most cases. ${ }^{25}$ p63, MOC-31 and TFT1 staining less than $10 \%$ of the control group of 73 various primary and metastatic sarcomas, melanomas, and benign spindle cell lesions. ${ }^{23}$ Immunohistochemical staining using a combination of TTF-1, napsin A, p63, and CK5/6 allows subclassification of poorly differentiated non-small cell lung carcinomas (NSCLCs) on small lung biopsies in most cases. Positive expression to TTF1 in biopsies from 16 of 20 adenocarcinomas (ACs); to p63 in all 15 squamous cell carcinomas (SCCs); to CK5/6 in 11 of 15 SCCs (all p63 positive) was registered. Staining with CK7 and CK20 antibodies can help discriminate between primary lung carcinoma and metastatic lung carcinoma. ${ }^{12}$ Staining for CK7 was present in 19 of $19 \mathrm{ACs}$ and 9 of $15 \mathrm{SCCs}^{28}$ The distinction between a large cell carcinoma and a sarcomatoid carcinoma is based on the perception of epithelial differentiation features. ${ }^{27}$ Pleomorphic leiomiosarcoma is characterized by smooth muscle differentiation, and pleomorphic liposarcoma with lipoblasts, often S100 + or Smooth Muscle Actin $(\mathrm{SMA})+.{ }^{17}$

Complex treatment: Surgical resection is the treatment of choice. ${ }^{4}$ In cases where the tumor is localized, surgery is an adequate course for treatment. Complete tumor excision in the early stages with proper follow-up care and perioperative chemotherapy are common therapies bringing satisfactory results. ${ }^{29}$ For patients with PSC, eligibility for surgical treatment, neoadjuvant or adjuvant chemotherapy based on the patient's ECOG score assessment can also be applied. ${ }^{30}$ Adjuvant chemotherapy and/or targeted therapy are recommended after surgery. ${ }^{5}$ Patients are almost always in the middle or advanced stages when they are diagnosed; these patients often do not have the opportunity to undergo surgical treatment. ${ }^{31}$ Since no data are currently available for the metastatic disease, patients are treated as having non-small cell lung cancer. ${ }^{32}$ In recent years, considerable progress has been made in gene sequencing, targeted therapies, and immunotherapies. ${ }^{5}$

\section{Conclusion}

The pulmonary sarcomatoid carcinoma (PSC) is extremely rarely lung neoplasm and extremely malignant with high risk of distant haematogenic metastases. The PSC is interested in the need for strict pathohistological and immunohistochemical analysis, a difficult pathohistological differential diagnosis with other primary malignant lung tumors and the assessment of complex treatment. In cases where the tumor is localized, surgery is an adequate course for treatment. Adjuvant chemotherapy and/or targeted therapy are recommended after surgery. In clinical cases with a large inoperable primary lung tumor with distant adrenals and brain metastases, the forecast is poor as it is an extremely radio/chemoresistance tumor cells. In our clinical case, only radiotherapy for brain metastases was possible. In order to improve the healing results and survival of patients, early diagnosis is required at early stage with surgical treatment and subsequent adjuvant chemotherapy and targeted therapy after genetic analysis of surgery or biopsy tissue material.

\section{Acknowledgments}

None.

\section{Conflicts of interest}

Author declares there are no conflicts of interest.

\section{Funding}

None.

\section{References}

1. Koss M, Travis W, Moran C. Pulmonary sarcomas, blastomas, carcinosarcomas and teratomas. In: Hasleton PS, editor. Spencer's pathology of lung. 5th ed. New York: McGraw-Hill; 1996. p. 1092-1100.

2. Yendamuri S, Caty L, Pine M, et al. Outcomes of sarcomatoid carcinoma of the lung: A Surveillance, Epidemiology, and End Results Database analysis. Surgery. 2012;152(3):397-402.

3. Barnard WG. Embryoma of lung. Thorax. 1952;7:299-301.

4. Ramakant Dixit, Nalin Joshi, Lokendra Dave. Biphasic pulmonary blastoma: An unusual presentation with chest wall, rib, and pleural involvement. Lung India. 2014;31(1):87-89.

5. Xin Li , Di Wu, Hongyu Liu, et al. Pulmonary sarcomatoid carcinoma: progress, treatment and expectations. Ther Adv Med Oncol. 2020;12:1758835920950207.

6. Ram R, Saadat P, Peng D, et al. Case report and literature review: Primary cutaneous carcinosarcoma. Ann Clin Lab Sci. 2005;35(2):189-94.

7. Jain D. Pleomorphic. Pathology Outlines. 2021.

8. Phatak AM, Mandke JV, Udani RH, et al. Pulmonary blastoma. Lung India. 1989;7:137-139.

9. Ashif Jethava, Constantin A Dasanu. Adult biphasic pulmonary blastoma. Conn Med. 2013;77(1):19-22.

10. Huang SY, Shen SJ, Li XY. Pulmonary sarcomatoid carcinoma: A clinicopathologic study and prognostic analysis of 51 cases. World J Surg Oncol. 2013;11:252.

11. Koss MN, Hochholzer L, O’Leary T. Pulmonary blastomas. Cancer. 1991;67:2368-81

12. Hafiza Sobia Arshad, Rizwan Ahmed Dudekula, Masooma Niazi, et al. A Rare Case of Sarcomatoid Carcinoma of the Lung with Spine Metastasis, Including a Literature Review. Am J Case Rep. 2017;18:760-765.

13. Ro JY, Chen JL, Lee JS, et al. Sarcomatoid carcinoma of the lung. Immunohistochemical and ultrastructural studies of 14 cases. Cancer. 1992;69(2):376-386.

14. Mark R Wick, Mark H Stoler. Sarcomas and Sarcomatoid Neoplasms of the Lungs and Pleural Surfaces in Practical Pulmonary Pathology: A Diagnostic Approach (Second Edition). 2012.

15. Baldovini C, Rossi G, Ciarrocchi A. Approaches to tumor classification in pulmonary sarcomatoid carcinoma. Lung Cancer (Auckl). 2019;10:131149.

16. Kitazawa R, Kitazawa S, Nishimura Y, et al. Lung carcinosarcoma with liposarcoma element: autopsy case. Pathol Int. 2006;56:449-452.

17. Shankar V. Undifferentiated / unclassified sarcoma. PathologyOutlines. 2021. 
18. Melora D Berardo, Celeste N Powers, Paul E Wakely, et al. Fine-needle aspiration cytopathology of malignant fibrous histiocytoma. Cancer (Cancer Cytopathol). 1997;81:228-237.

19. Samuel P Hammar, Sanja Dacic. Immunohistology of lung and pleural neoplasms. Sarcomoid carcinoma. In Diagnostic Immunohistochemistry (Third Edition). 2011.

20. Kim MJ, Shin HC, Shin KC. Best immunohistochemical panel in distinguishing adenocarcinoma from squamous cell carcinoma of lung: Tissue microarray assay in resected lung cancer specimens. Ann Diagn Pathol. 2013;17(1):85-90.

21. Amir Qorbani, Scott D Nelson. Primary pulmonary undifferentiated pleomorphic sarcoma (PPUPS). Autops Case Rep. 2019;9(3):e2019110.

22. Addis BJ, Corrin B. Pulmonary blastoma, carcinosarcoma and spindlecell carcinoma: An immunohistochemical study of keratin intermediate filaments. J Pathol. 1985;147(4):291-301.

23. James S Lewis, Jon H Ritter, Samir El-Mofty. Alternative epithelial markers in sarcomatoid carcinomas of the head and neck, lung, and bladder-p63, MOC-31, and TTF-1. Mod Pathol. 2005;18(11):1471-1481.

24. Hansen T, Bittinger F, Kortsik C, et al. Expression of KIT (CD117) in biphasic pulmonary blastoma. Novel data on histogenesis. Lung. 2003;181:193-200.

25. Artem Smirnov, Lucia Anemona, Flavia Novelli, et al. p63 Is a Promising Marker in the Diagnosis of Unusual Skin Cancer. Int $\mathrm{J} \mathrm{Mol} \mathrm{Sci.}$ 2019;20(22):5781.
26. Moses M A, George A L, Sakakibara N, et al. Molecular Mechanisms of P63-Mediated Squamous Cancer Pathogenesis. Int $\mathrm{J} \mathrm{Mol} \mathrm{Sci}$. 2019;20:3590.

27. Alberto M, Marchevsky. Pathologic classification of lung malignancies and special pathologic procedures in medical management of the thoracic surgery patient, 2010

28. Sanjay Mukhopadhyay, Anna-Luise A Katzenstein. Subclassification of non-small cell lung carcinomas lacking morphologic differentiation on biopsy specimens: Utility of an immunohistochemical panel containing TTF-1, napsin A, p63, and CK5/6. Am J Surg Pathol. 2011;35(1):15-25.

29. . Kaira K, Horie Y, Ayabe E, et al. Pulmonary pleomorphic carcinoma: a clinicopathological study including EGFR mutation analysis. $J$ Thorac Oncol. 2010;5:460-465.

30. Chaft JE, Sima CS, Ginsberg MS, et al. Clinical outcomes with perioperative chemotherapy in sarcomatoid carcinomas of the lung. $J$ Thorac Oncol. 2012;7:1400-1405.

31. Hou J, Xing L, Yuan Y. A clinical analysis of 114 cases of sarcomatoid carcinoma of the lung. Clin Exp Med. 2018;18(4):555-562.

32. Mason RJ, Broaddus VC, Martin T, et al. Murray and Nadel's Textbook of Respiratory Medicine. 5th ed. 2010. Rare malignant primary pulmonary epithelial tumors. Ch. 48, Part 4. Primary Pulmonary Sarcomas. 